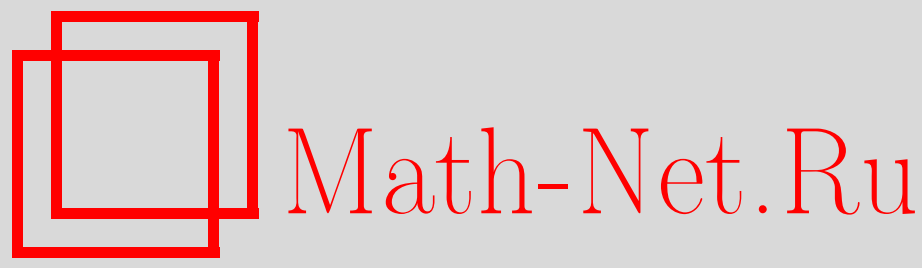

И. В. Чередник, Неотрицательный базис решетки, Дискрет. матем., 2014, том 26, выпуск 3, 127-135

DOI: https://doi.org/10.4213/dm1296

Использование Общероссийского математического портала Math-Net.Ru подразумевает, что вы прочитали и согласны с пользовательским соглашением http://www . mathnet.ru/rus/agreement

Параметры загрузки:

IP : 54.209 .52 .79

26 апреля 2023 г., 03:12:53

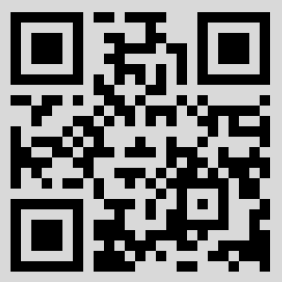




\title{
Неотрицательный базис решетки
}

\author{
() 2014 г. И. В. Чередник*
}

Строится неотрицательный базис полной решетки. Доказывается критерий существования неотрицательного базиса у произвольной решетки. Исследуется вопрос о существовании базиса решетки в произвольном выпуклом конусе.

Ключевые слова: неотрицательный базис решетки, целочисленная решетка, выпуклый конус.

\section{Введение}

Один из наиболее распространенных способов построения псевдослучайных последовательностей связан с использованием линейных и полилинейных рекуррентных последовательностей над конечными модулями $[1,2]$. При этом изучение периодических свойств линейных и полилинейных рекуррент является первоочередной задачей, поскольку период - одна из важнейших характеристик псевдослучайной последовательности. С этой целью в работах $[1,2]$ вводится и исследуется понятие группы вектор-периодов последовательности.

Понятие группы вектор-периодов тесно связано с решетками, а именно, группа вектор-периодов является подгруппой группы $\mathbb{Z}^{n}$ (целочисленной решеткой) ранга $n$ и порождается всеми своими неотрицательными элементами (векторами с неотрицательными координатами), причем данное свойство является характеристическим: любая подгруппа $\mathbb{Z}^{n}$ с указанным свойством является группой вектор-периодов некоторой полилинейной последовательности [2]. В связи с этим авторами $[1,2]$ поставлен вопрос об описании всех подгрупп $\mathbb{Z}^{n}$ с таким свойством.

В данной работе показано, что каждая подгруппа $\mathbb{Z}^{n}$ ранга $n$ порождается множеством всех своих неотрицательных векторов, более того, строятся неотрицательные базисы таких групп. Эти результаты удается обобщить на случай произвольной полной решетки евклидова пространства. Также в работе доказывается, что необходимым и достаточным условием наличия неотрицательного базиса у произвольной решетки евклидова пространства является наличие неотрицательной системы образующих. Дальнейшим развитием данного исследования стало построение базиса решетки евклидова пространства в произвольном выпуклом конусе.

\section{1. Основные определения}

Пусть $\mathbb{R}^{n}$ - евклидово пространство размерности $n$, в котором скалярное произведение пары векторов $\alpha=\left(a_{1}, a_{2}, \ldots, a_{n}\right), \beta=\left(b_{1}, b_{2}, \ldots, b_{n}\right)$ определено по правилу

$$
(\alpha, \beta)=a_{1} b_{1}+a_{2} b_{2}+\ldots+a_{n} b_{n} .
$$

* Место работы: Лаборатория ТВП, e-mail: p.n.v.k.s@mail.ru 
Решеткой размерности $k$ в пространстве $\mathbb{R}^{n}$ называется любая подгруппа $L$ группы $\left(\mathbb{R}^{n},+\right)$, порожденная линейно независимой системой векторов $\beta_{1}, \beta_{2}, \ldots, \beta_{k}$ из $\mathbb{R}^{n}$, которую называют базисом решетки. Если $k=n$, то решетка называется полной. $\mathrm{B}$ случае, когда $\beta_{1}, \beta_{2}, \ldots, \beta_{k} \in \mathbb{Z}^{n}$, решетка называется целочисленной.

Поскольку решетки являются конечно-порожденными свободными абелевыми группами, нам будет удобно использовать общепринятую групповую терминологию. Так, например, мощность базиса решетки в групповой терминологии называется рангом группы.

Множество

$$
\Pi_{\beta_{1}, \ldots, \beta_{k}}=\left\{x_{1} \beta_{1}+x_{2} \beta_{2}+\ldots+x_{k} \beta_{k} \mid 0 \leqslant x_{i} \leqslant 1, i=1, \ldots, k\right\}
$$

называется фундаментальным или главным параллелепипедом решетки $L$ в базисе $\beta_{1}, \beta_{2}, \ldots, \beta_{k}$. Объем фундаментального параллелепипеда $\Pi_{\beta_{1}, \ldots, \beta_{k}}$ решетки $L$ можно вычислить по формуле

$$
\sqrt{\operatorname{det}\left(G\left(\beta_{1}, \beta_{2}, \ldots, \beta_{k}\right)\right)}
$$

где $G\left(\beta_{1}, \beta_{2}, \ldots, \beta_{k}\right)$ - матрица Грама системы векторов $\beta_{1}, \beta_{2}, \ldots, \beta_{k}$. Поскольку объем фундаментального параллелепипеда решетки $L$ не зависит от выбора базиса (см. [3]), то данную величину корректно называть обгемом или определителем решетки $L$.

\section{2. Неотрицательный базис решетки}

Вектор $\alpha=\left(a_{1}, a_{2}, \ldots, a_{n}\right) \in \mathbb{R}^{n}$ будем называть неотрицательным и использовать запись $\alpha \geqslant 0$, если $a_{i} \geqslant 0$ для всех $i \in\{1, \ldots, n\}$.

Предложение 1. Полная целочисленная решетка $L \subset \mathbb{R}^{n}$ обладает неотрицательным базисом.

Доказательство. Доказательство проведем индукцией по параметру $n$. При $n=1$ любая подгруппа $(\mathbb{Z},+)$ имеет вид $d \mathbb{Z}$, и утверждение очевидно.

Пусть $N>1$ и утверждение верно при всех $n<N$. Докажем его при $n=N$. Рассмотрим проекцию группы $L$ на первую координату:

$$
L_{1}=\{a \in \mathbb{Z} \mid \exists(a, *, \ldots, *) \in L\} .
$$

Из известного описания подгрупп $(\mathbb{Z},+)$ следует, что $L_{1}=d \mathbb{Z}$. Значит, существует $\gamma_{1} \in L: \gamma_{1}=(d, *, \ldots, *)$. При этом $d>0$, так как ранг $L$ равен $n$.

Рассмотрим теперь множество всех векторов из $L$, у которых первая координата равна нулю:

$$
L_{0}=\{(0, *, \ldots, *) \in L\} .
$$

Легко понять, что $L_{0}$ является группой ранга $n-1$ и $L=\left\langle\gamma_{1}\right\rangle \dot{+} L_{0}$. При этом $L_{0}$ изоморфна соответствующей подгруппе $M$ группы $\left(\mathbb{Z}^{n-1},+\right)$ (достаточно вычеркнуть у всех векторов из $L_{0}$ первую координату). По предположению индукции в группе $M$ существует неотрицательный базис $\gamma_{2}, \ldots, \gamma_{n}$, а значит, и группа $L_{0}$ обладает неотрицательным базисом: $\alpha_{2}=\left(0, \gamma_{2}\right), \ldots, \alpha_{n}=\left(0, \gamma_{n}\right)$.

Так как ранг группы $L_{0}$ равен $n-1$, то невозможно, чтобы какая-либо координата, кроме первой, была нулевой у всех ее базисных векторов $\alpha_{2}, \ldots, \alpha_{n}$. Значит, подобрав достаточно большие числа $b_{2}, \ldots, b_{n} \in \mathbb{N}$, можно получить неотрицательный вектор:

$$
\alpha_{1}=\gamma_{1}+b_{2} \alpha_{2}+\ldots+b_{n} \alpha_{n}=(d, *, \ldots, *) \geqslant 0
$$


Несложно понять, что система неотрицательных векторов $\alpha_{1}, \alpha_{2}, \ldots, \alpha_{n}$ является базисом группы $L$.

В работе [2] (стр. 164, предложение 3.5) доказано характеристическое свойство групп вектор-периодов: подгруппа $\left(\mathbb{Z}^{n},+\right)$ ранга $n$ является группой вектор-периодов некоторой полилинейной последовательности в том и только в том случае, когда она порождается множеством всех своих неотрицательных элементов. Воспользовавшись этой характеристикой, мы получаем полное описание групп вектор-периодов.

Следствие 1. Любая подгруппа $\left(\mathbb{Z}^{n},+\right)$ ранга $n$ порождается множеством всех своих неотрицательных векторов, и является группой вектор-периодов некоторой полилинейной последовательности.

Несложно понять, что в случае, когда полная решетка $L=\left\langle\beta_{1}, \beta_{2}, \ldots, \beta_{n}\right\rangle_{\mathbb{Z}}$ является дробно-рациональной, то есть $L \subset \mathbb{Q}^{n}$, построение неотрицательного базиса решетки $L$ естественным образом сводится к построению неотрицательного базиса подходящей целочисленной решетки $d L=\left\langle d \beta_{1}, d \beta_{2}, \ldots, d \beta_{n}\right\rangle_{\mathbb{Z}} \subset \mathbb{Z}^{n}$. Дальнейшее обобщение предложения 1 на случай произвольной полной решетки $L=\left\langle\beta_{1}, \beta_{2}, \ldots, \beta_{n}\right\rangle_{\mathbb{Z}} \subset \mathbb{R}^{n}$ достигается путем «аппроксимации» вещественного базиса $\beta_{1}, \beta_{2}, \ldots, \beta_{n}$ дробным $\beta_{1}^{\prime}, \beta_{2}^{\prime}, \ldots, \beta_{n}^{\prime}$ и переходом к соответствующей дробно-рациональной решетке $L^{\prime}=\left\langle\beta_{1}^{\prime}, \beta_{2}^{\prime}, \ldots, \beta_{n}^{\prime}\right\rangle_{\mathbb{Z}}$.

Теорема 1. Любая полная решетка $L \subset \mathbb{R}^{n}$ имеет неотрицательный базис.

Доказательство. Докажем предварительно следующее вспомогательное утверждение.

Лемма 1. Пусть $A_{n \times n}$ - невырожденная матрица над $\mathbb{R}$. Тогда существуют такие целочисленная матрица $B_{n \times n}$ и неотрицательная матрица $H_{n \times n}$, что $A=B \cdot H$.

Доказательство. Пусть $A=\left(\begin{array}{llll}\alpha_{1}^{\downarrow} & \alpha_{2}^{\downarrow} & \ldots & \alpha_{n}^{\downarrow}\end{array}\right)$. Для доказательства леммы построим две последовательности матриц $A_{0}=A, A_{1}, A_{2}, \ldots, A_{n}$ и $H_{1}, H_{2}, \ldots, H_{n}$ со свойствами:

1. $A_{i}=\left(\begin{array}{lllll}\beta_{1}^{\downarrow} & \ldots & \beta_{i}^{\downarrow} & \alpha_{i+1}^{\downarrow} \ldots & \alpha_{n}^{\downarrow}\end{array}\right), \beta_{1}^{\downarrow}, \ldots, \beta_{i}^{\downarrow}$ - целочисленные векторы;

2. $H_{1}, H_{2}, \ldots, H_{n}$ - неотрицательные матрицы;

3. $A_{i-1}=A_{i} \cdot H_{i}$ для $i=1, \ldots, n$. Несложно понять, что матрицы $B=A_{n}$ и $H=H_{n} \cdot H_{n-1} \cdots \cdot H_{1}$ будут являться подходящими целочисленной и неотрицательной матрицами:

$$
A=A_{n} \cdot H_{n} \cdot H_{n-1} \cdot \ldots \cdot H_{1} .
$$

Рассмотрим множество $M=\left\{c_{1} \alpha_{1}^{\downarrow}+c_{2}\left(-\alpha_{2}^{\downarrow}\right)+\ldots+c_{n}\left(-\alpha_{n}^{\downarrow}\right) \mid c_{i} \in \mathbb{R}_{>0}, i=1, \ldots, n\right\}$, состоящее из всех таких векторов $\beta^{\downarrow}$ пространства $\mathbb{R}^{n}$, что вектор $\alpha_{1}^{\downarrow}$ можно представить в виде положительной линейной комбинации векторов $\beta^{\downarrow}, \alpha_{2}^{\downarrow}, \ldots, \alpha_{n}^{\downarrow}$. Нетрудно проверить, что множество $M$ является открытым в пространстве $\mathbb{R}^{n}$, то есть вместе с каждым элементом $\beta^{\downarrow} \in M$ в множестве $M$ содержится некоторая его окрестность $\mathrm{U}_{\beta \downarrow}$. В силу плотности множества $\mathbb{Q}^{n}$ в $\mathbb{R}^{n}$ из окрестности $\mathrm{U}_{\beta \downarrow} \subset M$ можно выбрать дробно-рациональный вектор $\beta^{\prime \downarrow} \in \mathbb{Q}^{n}$, и представить вектор $\alpha_{1}^{\downarrow}$ виде положительной линейной комбинации векторов $\beta^{\prime \downarrow}, \alpha_{2}^{\downarrow}, \ldots, \alpha_{n}^{\downarrow}$ :

$$
\alpha_{1}^{\downarrow}=d^{\prime} \beta^{\prime \downarrow}+d_{2} \alpha_{2}^{\downarrow}+\ldots+d_{n} \alpha_{n}^{\downarrow} .
$$


Очевидно, что в полученной линейной комбинации дробно-рациональный вектор $\beta^{\prime}$ можно заменить на такой целочисленный $\beta_{1}^{\downarrow}=k \beta^{\prime \downarrow}$, что линейная комбинация

$$
\alpha_{1}^{\downarrow}=d_{1} \beta_{1}^{\downarrow}+d_{2} \alpha_{2}^{\downarrow}+\ldots+d_{n} \alpha_{n}^{\downarrow}
$$

останется положительной. Легко заметить, что указанная линейная комбинация определяет матрицу $A_{1}=\left(\begin{array}{llll}\beta_{1}^{\downarrow} & \alpha_{2}^{\downarrow} & \ldots & \alpha_{n}^{\downarrow}\end{array}\right)$, где $\beta_{1}^{\downarrow}$ - целочисленный вектор, и неотрицательную матрицу $H_{1}$ :

$$
\left(\begin{array}{cccc}
d_{1} & 0 & \ldots & 0 \\
d_{2} & 1 & \ldots & 0 \\
\vdots & \vdots & \ddots & \vdots \\
d_{n} & 0 & \ldots & 1
\end{array}\right)
$$

и при этом $A=A_{1} \cdot H_{1}$. Остальные матрицы последовательностей $A_{1}, A_{2}, \ldots, A_{n}$ и $H_{1}, H_{2}, \ldots, H_{n}$ строятся аналогично.

Пусть $\alpha_{1}, \alpha_{2}, \ldots, \alpha_{n} \in \mathbb{R}^{n}$ - произвольный базис решетки $L \subset \mathbb{R}^{n}$. Обозначим через $A$ квадратную матрицу, составленную из векторов $\alpha_{1}, \alpha_{2}, \ldots, \alpha_{n}$. Согласно лемме 1 существуют такие целочисленная матрица $B$ и неотрицательная матрица $H$, что выполняется равенство $A=B \cdot H$ :

$$
\left(\begin{array}{l}
\overrightarrow{\alpha_{1}} \\
\overrightarrow{\alpha_{2}} \\
\cdots \\
\overrightarrow{\alpha_{n}}
\end{array}\right)=\left(\begin{array}{l}
\overrightarrow{\beta_{1}} \\
\overrightarrow{\beta_{2}} \\
\cdots \\
\overrightarrow{\beta_{n}}
\end{array}\right) \cdot H
$$

По предложению 1 полная целочисленная решетка, порожденная векторами $\beta_{1}, \beta_{2}, \ldots, \beta_{n}$, обладает неотрицательным базисом. Целочисленную матрицу перехода к этому неотрицательному базису обозначим через $Z$. Тогда из соотношения (1) получаем, что система векторов $\gamma_{1}, \gamma_{2}, \ldots, \gamma_{n}$, определяемая равенствами

$$
\left(\begin{array}{l}
\overrightarrow{\gamma_{1}} \\
\overrightarrow{\gamma_{2}} \\
\cdots \\
\overrightarrow{\gamma_{n}}
\end{array}\right)=Z \cdot\left(\begin{array}{l}
\overrightarrow{\alpha_{1}} \\
\overrightarrow{\alpha_{2}} \\
\ldots \\
\overrightarrow{\alpha_{n}}
\end{array}\right)=Z \cdot\left(\begin{array}{l}
\overrightarrow{\beta_{1}} \\
\overrightarrow{\beta_{2}} \\
\ldots \overrightarrow{\beta_{n}}
\end{array}\right) \cdot H=Z \cdot B \cdot H,
$$

является неотрицательным базисом решетки $L$, поскольку матрица $Z$ - обратимая и целочисленная, а $Z \cdot B$ и $H$ - неотрицательные матрицы.

Следует отметить, что не все решетки обладают неотрицательным базисом. Например, группа, порожденная $n-1$ векторами

$$
\langle(1,-1,0, \ldots, 0),(0,0,1, \ldots, 0), \ldots,(0,0, \ldots, 0,1)\rangle_{\mathbb{Z}} \in \mathbb{R}^{n}
$$

не порождается даже множеством всех своих неотрицательных элементов. Следующая теорема устанавливает прямую связь между этими двумя свойствами.

Предложение 2. Если решетка $L \subset \mathbb{R}^{n}$ порождается множеством всех своих неотрицательных векторов, то она имеет неотрицательный базис. 
Доказательство. Из условия несложно понять, что у группы $L$ существует конечная система образующих $\alpha_{1}, \alpha_{2}, \ldots, \alpha_{m}$, состоящая из неотрицательных векторов (достаточно выбрать множество неотрицательных векторов, порождающих некоторый базис группы $L$ ). Пусть $\alpha_{i_{1}}, \ldots, \alpha_{i_{k}}$ - наибольшая линейно независимая подсистема векторов. Тогда выполняются некоторые целочисленные соотношения:

$$
\begin{gathered}
c_{i_{1} 1} \alpha_{i_{1}}+\ldots+c_{i_{k} 1} \alpha_{i_{k}}=d_{1} \alpha_{1}, \\
c_{i_{1} 2} \alpha_{i_{1}}+\ldots+c_{i_{k} 2} \alpha_{i_{k}}=d_{2} \alpha_{2}, \\
\ldots \\
c_{i_{1} m} \alpha_{i_{1}}+\ldots+c_{i_{k} m} \alpha_{i_{k}}=d_{m} \alpha_{m} .
\end{gathered}
$$

Рассмотрим систему неотрицательных векторов $\gamma_{1}, \gamma_{2}, \ldots, \gamma_{k} \in \mathbb{R}^{n}$, определенную следующим образом:

$$
\gamma_{1}=\frac{\alpha_{i_{1}}}{\left|d_{1} \cdot d_{2} \cdot \ldots \cdot d_{m}\right|}, \gamma_{2}=\frac{\alpha_{i_{2}}}{\left|d_{1} \cdot d_{2} \cdot \ldots \cdot d_{m}\right|}, \ldots, \gamma_{k}=\frac{\alpha_{i_{k}}}{\left|d_{1} \cdot d_{2} \cdot \ldots \cdot d_{m}\right|}
$$

Нетрудно видеть, что каждый вектор из системы $\alpha_{1}, \alpha_{2}, \ldots, \alpha_{m}$ является целочисленной линейной комбинацией неотрицательных векторов $\gamma_{1}, \gamma_{2}, \ldots, \gamma_{k}$, а значит, и каждый вектор решетки $L$ является целочисленной линейной комбинацией неотрицательных векторов $\gamma_{1}, \gamma_{2}, \ldots, \gamma_{k}$. Таким образом, $L-$ подгруппа свободной абелевой группы $\left\langle\gamma_{1}, \gamma_{2}, \ldots, \gamma_{k}\right\rangle_{\mathbb{Z}}$, и при этом их ранги совпадают.

При естественном изоморфизме свободных абелевых групп

$$
\left\langle\gamma_{1}, \gamma_{2}, \ldots, \gamma_{k}\right\rangle_{\mathbb{Z}} \longrightarrow \mathbb{Z}^{k}: \gamma_{i} \mapsto(0, \ldots, 1, \ldots, 0)
$$

образ решетки $L$ является полной решеткой в $\mathbb{Z}^{k}$, и по предложению 1 обладает неотрицательным базисом:

$$
\left(b_{11}, \ldots, b_{1 k}\right),\left(b_{21}, \ldots, b_{2 k}\right), \ldots \ldots,\left(b_{k 1}, \ldots, b_{k k}\right) .
$$

Из свойств изоморфизма следует, что неотрицательная система векторов

$$
\begin{gathered}
b_{11} \gamma_{1}+b_{12} \gamma_{2}+\ldots+b_{1 k} \gamma_{k} \\
b_{21} \gamma_{1}+b_{22} \gamma_{2}+\ldots+b_{2 k} \gamma_{k} \\
\ldots \\
b_{k 1} \gamma_{1}+b_{k 2} \gamma_{2}+\ldots+b_{k k} \gamma_{k}
\end{gathered}
$$

является базисом $L$.

Следствие 2. Решетка $L \subset \mathbb{R}^{n}$ имеет неотрицательный базис тогда и только тогда, когда она порождается множеством всех своих неотрицательных векторов.

\section{3. Базис решетки из выпуклого конуса}

Подмножество $M$ в $\mathbb{R}^{n}$ называется выпуклым, если $(1-a) \alpha+a \beta \in M$ для любых $\alpha \in$ $M, \beta \in M$ и $0<a<1$. Важный пример выпуклых множеств - полупространства. Для ненулевого элемента $\beta \in \mathbb{R}^{n}$ и любого $b \in \mathbb{R}$ множества

$$
\left\{\alpha \in \mathbb{R}^{n} \mid(\alpha, \beta) \geqslant b\right\}, \quad\left\{\alpha \in \mathbb{R}^{n} \mid(\alpha, \beta) \leqslant b\right\}
$$


называются замкнутыми полупространствами. Множества

$$
\left\{\alpha \in \mathbb{R}^{n} \mid(\alpha, \beta)>b\right\}, \quad\left\{\alpha \in \mathbb{R}^{n} \mid(\alpha, \beta)<b\right\}
$$

называются открытыми полупространствами. Легко проверить, что пересечение любого числа выпуклых множеств выпукло. Следовательно, множество

$$
\left\{\alpha \in \mathbb{R}^{n} \mid\left(\alpha, \beta_{i}\right) \geqslant b_{i} \forall i \in I\right\}
$$

тоже является выпуклым. Множество, которое может быть представлено как пересечение конечного числа полупространств в $\mathbb{R}^{n}$, называется полиэдралъным выпуклым множеством, или выпуклым полиэдром. Иногда, для удобства, конечную систему неравенств, задающих выпуклый полиэдр, будем записывать в матричной форме: $\left(x_{1}, x_{2}, \ldots, x_{n}\right) B_{n \times m} \geqslant\left(b_{1}, b_{2}, \ldots, b_{m}\right)$.

Размерностъю выпуклого множества $M$ называется размерность его аффинной оболочки - минимального линейного многообразия, содержащего множество $M$. Напомним, что размерностью многообразия считается размерность параллельного ему подпространства.

Рассмотрим важный класс выпуклых множеств, обладающих свойством неограниченности. Подмножество $K \subset \mathbb{R}^{n}$ называется конусом, если оно замкнуто относительно умножения на неотрицательные числа, иначе говоря, $a \alpha \in K$ для всех $\alpha \in K$ и $a \geqslant 0$. Такое множество есть объединение лучей, выходящих из начала координат. Конус не обязательно "заострен", например, всякое подпространство в $\mathbb{R}^{n}$ есть конус, открытое и замкнутое полупространства также являются конусами. Выпуклый конус - это конус, являющийся выпуклым множеством. Два наиболее важных выпуклых конуса - это неотрицателъный ортант в $\mathbb{R}^{n}$ :

$$
\left\{\alpha=\left(a_{1}, a_{2}, \ldots, a_{n}\right) \mid a_{1} \geqslant 0, a_{2} \geqslant 0, \ldots, a_{n} \geqslant 0\right\},
$$

и положительный ортант в $\mathbb{R}^{n}$ :

$$
\left\{\alpha=\left(a_{1}, a_{2}, \ldots, a_{n}\right) \mid a_{1}>0, a_{2}>0, \ldots, a_{n}>0\right\} .
$$

Легко проверяется, что пересечение любого числа выпуклых конусов является выпуклым конусом. Следовательно множество

$$
\left\{\alpha \in \mathbb{R}^{n} \mid\left(\alpha, \beta_{i}\right) \geqslant 0, i \in I\right\}
$$

есть выпуклый конус.

Пересечение всех выпуклых конусов, содержащих заданное множество $S$, называется выпуклым конусом, порожденным $S$, и обозначается сопе $S$. Если множество $S$ конечно, то cone $S$ называется конечнопорожденным выпуклым конусом с системой образующих $S$. Приведем без доказательства интересный результат, который будет использован в дальнейшем.

Теорема 2 [4; с. 188]. Конечнопорожденный выпуклый конус $K$ является выпуклым полиэдром, то есть задается конечной системой линейных неравенств.

В предыдущем разделе было доказано, что любая полная решетка в пространстве $\mathbb{R}^{n}$ имеет неотрицательный базис. Другими словами, любая полная решетка в $\mathbb{R}^{n}$ имеет базис в простейшем выпуклом конусе - неотрицательном $n$-мерном ортанте. Данный результат можно обобщить следующим образом. 
Теорема 3. Полная решетка $L \subset \mathbb{R}^{n}$ имеет базис в произвольном выпуклом конусе размерности $n$.

Доказательство. Пусть $K$ - выпуклый конус размерности $n$. Тогда, по определению, существует система линейно независимых векторов $\alpha_{1}, \alpha_{2}, \ldots, \alpha_{n} \in K$. Для доказательства теоремы достаточно показать, что решетка $L$ имеет базис в выпуклом множестве cone $\left\{\alpha_{1}, \alpha_{2}, \ldots, \alpha_{n}\right\} \subset K$. Как было отмечено ранее, множество cone $\left\{\alpha_{1}, \alpha_{2}, \ldots, \alpha_{n}\right\}$ является полиэдральным и может быть задано системой линейных неравенств $\left(x_{1}, x_{2}, \ldots, x_{n}\right) B_{n \times n} \geqslant(0,0, \ldots, 0)$, где $B-$ невырожденная матрица $([4])$.

Несложно понять, что невырожденное линейное преобразование $\mathbb{R}^{n}$, определенное по правилу

$$
\left(x_{1}, x_{2}, \ldots, x_{n}\right) \mapsto\left(x_{1}, x_{2}, \ldots, x_{n}\right) B,
$$

задает естественный изоморфизм решеток $L$ и $L_{B}=\{\alpha B \mid \alpha \in L\}$. Значит ранг группы $L_{B}$ равен $n$, и по теореме 1 у нее существует неотрицательный базис, который, в силу изоморфизма решеток, имеет вид $\gamma_{1} B, \gamma_{2} B, \ldots, \gamma_{n} B$, где $\gamma_{1}, \gamma_{2}, \ldots, \gamma_{n}$ - некоторый базис $L$. Легко видеть, что указанный базис группы $L$ удовлетворяет системе неравенств $\left(x_{1}, x_{2}, \ldots, x_{n}\right) B_{n \times n} \geqslant(0,0, \ldots, 0)$, а значит, содержится в cone $\left\{\alpha_{1}, \alpha_{2}, \ldots, \alpha_{n}\right\} \subset K$.

Следствие 3. Полная решетка $L \subset \mathbb{R}^{n}$ имеет положительный базис.

Теорема 3 обладает интересной геометрической интерпретацией. Ее формулировку можно трактовать следующим образом: полная решетка обладает фундаментальным параллелепипедом в любом конусе максимальной размерности. Так как конус можно выбрать в любом «направлении» и сколь угодно «узким», то можно считать, что полная решетка обладает сколь угодно «узким» фундаментальным параллелепипедом в любом «направлении». При этом, как известно, объем фундаментального параллелепипеда решетки - величина, не зависящая от выбора базиса.

Предложение 3. Если решетка $L \subset \mathbb{R}^{n}$ порождается множеством своих элементов из некоторого выпуклого конуса $K$, то она имеет базис в $K$.

Доказательство. Пусть $k$ - размерность решетки $L$. Рассмотрим произвольное линейное отображение $\varphi: \mathbb{R}^{n} \longrightarrow \mathbb{R}^{k}$, при котором образом решетки $L$ является полная решетка $\mathbb{Z}^{k}$. Несложно понять, что образом выпуклого конуса $K$ при линейном отображении $\varphi$ будет тоже выпуклый конус $\varphi(K)$. При этом, поскольку $K$ содержит систему образующих решетки $L$, конус $\varphi(K)$ содержит систему образующих решетки $\mathbb{Z}^{k}$, и значит, его размерность равна $k$.

По теореме 3 решетка $\mathbb{Z}^{k}$ обладает базисом в выпуклом конусе $\varphi(K)$ :

$$
\varphi\left(\gamma_{1}\right), \varphi\left(\gamma_{2}\right), \ldots, \varphi\left(\gamma_{k}\right)
$$

где $\gamma_{1}, \gamma_{2}, \ldots, \gamma_{k} \in K$. Очевидно, что $\varphi$ задает изоморфизм свободных групп $L$ и $\mathbb{Z}^{k}$, а значит $\gamma_{1}, \gamma_{2}, \ldots, \gamma_{k} \in K-$ искомый базис решетки $L$.

Следствие 4. Решетка имеет базис в выпуклом конусе в том и только в том случае, когда она порождается своими элементами из этого конуса.

Для $M \subset \mathbb{R}^{n}$ и $\alpha \in \mathbb{R}^{n}$ сдвигом, или транслянтом множества $M$ на вектор $\alpha$ (короче, $\alpha$-транслянтом множества $M$ ) называется множество

$$
M+\alpha=\{\mu+\alpha \mid \mu \in M\} .
$$


Легко видеть, что транслянт выпуклого множества есть снова выпуклое множество, притом такой же размерности.

Теорема 4. Полная решетка $L \subset \mathbb{R}^{n}$ имеет базис в любом транслянте выпуклого конуса размерности $n$.

Доказательство. Пусть $K \subset \mathbb{R}^{n}$ - выпуклый конус размерности $n$, а $\alpha+K, \alpha=$ $\left(a_{1}, \ldots, a_{n}\right) \in \mathbb{R}^{n},-$ его произвольный сдвиг. В доказательстве теоремы 3 было построено полиэдральное множество $C \subset K$, которое определяется системой линейных неравенств

$$
\left(x_{1}, x_{2}, \ldots, x_{n}\right) B_{n \times n} \geqslant(0,0, \ldots, 0), \quad B \in \mathbb{R}_{n, n}^{*},
$$

и указан базис $\gamma_{1}, \gamma_{2}, \ldots, \gamma_{n}$ решетки $L$, содержащийся в $C$.

Несложно проверить, что $\alpha$-транслянт множества $C$ также является полиэдральным множеством и при этом задается системой линейных неравенств

$$
\left(x_{1}-a_{1}, x_{2}-a_{2}, \ldots, x_{n}-a_{n}\right) B_{n \times n} \geqslant(0,0, \ldots, 0),
$$

или, что равносильно

$$
\left(x_{1}, x_{2}, \ldots, x_{n}\right) B_{n \times n} \geqslant\left(c_{1}, c_{2}, \ldots, c_{n}\right),
$$

где $\left(c_{1}, c_{2}, \ldots, c_{n}\right)=\left(a_{1}, a_{2}, \ldots, a_{n}\right) B$.

Таким образом, для доказательства текущей теоремы достаточно будет показать, что у решетки $L$ существует базис, содержащийся в множестве $\alpha+C \subset \alpha+K$.

Пусть $i$ - наименьший номер неравенства в системе (3), которому не удовлетворяет хотя бы один из векторов базиса $\gamma_{1}, \gamma_{2}, \ldots, \gamma_{n}$. При этом каждый вектор из указанного базиса удовлетворяет $i$-му неравенству системы $(2)$, и найдется хотя бы один вектор $\gamma_{j}$, для которого $i$-е неравенство системы (2) выполняется строго: $\left(\gamma_{j},\left(b_{1 i}, b_{2 i}, \ldots, b_{n i}\right)\right)=d>0$ (в противном случае $i$-е неравенство будет тривиальным, а матрица $B$ - вырожденной). Тогда в базисе

$$
\gamma_{1}+\left\lceil\frac{c_{i}}{d}\right\rceil \gamma_{j}, \ldots, \gamma_{j-1}+\left\lceil\frac{c_{i}}{d}\right\rceil \gamma_{j}, \gamma_{j}, \gamma_{j+1}+\left\lceil\frac{c_{i}}{d}\right\rceil \gamma_{j}, \ldots, \gamma_{n}+\left\lceil\frac{c_{i}}{d}\right\rceil \gamma_{j}
$$

для каждого вектора, кроме быть может $\gamma_{j}$, гарантированно выполняется $i$-е неравенство системы (3). А в базисе

$$
\gamma_{1}+\left\lceil\frac{c_{i}}{d}\right\rceil \gamma_{j}, \ldots, \gamma_{j-1}+\left\lceil\frac{c_{i}}{d}\right\rceil \gamma_{j}, \gamma_{j-1}+\left(\left\lceil\frac{c_{i}}{d}\right\rceil+1\right) \gamma_{j}, \gamma_{j+1}+\left\lceil\frac{c_{i}}{d}\right\rceil \gamma_{j}, \ldots, \gamma_{n}+\left\lceil\frac{c_{i}}{d}\right\rceil \gamma_{j}
$$

вообще для каждого вектора выполняется $i$-е неравенство системы (3). При этом, если все векторы исходного базиса $\gamma_{1}, \gamma_{2}, \ldots, \gamma_{n}$ удовлетворяют некоторому неравенству из системы (3), то и все векторы нового базиса (как натуральные линейные комбинации векторов исходного) также будут удовлетворять этому неравенству.

Таким образом, итеративно применив описанные выше действия к каждому неравенству системы (3), мы получим базис $\gamma_{1}^{*}, \gamma_{2}^{*}, \ldots, \gamma_{n}^{*}$ решетки $L$, удовлетворяющий системе неравенств (3), то есть содержащийся в $\alpha$-транслянте $C$.

Следует отметить, что предложение 3 и следствие 4 , в отличие от теоремы 3 , не обобщаются на случай произвольного сдвига выпуклого конуса, поскольку пересечение произвольной решетки с транслянтом конуса, вообе говоря, может быть конечным множеством. А из конечной системы образующих решетки не всегда зможно выделить ее базис.

Автор признателен А. А. Нечаеву за поставленную первоначальную задачу. 


\section{Список литературы}

1. Kurakin V. L., Kuzmin A. S., Mikhalev A. V., Nechaev A. A., "Linear recurring sequences over rings and modules.", J. Math. Sciences, 76:6 (1995), 2793-2915.

2. Кузьмин А. С., Куракин В. Л., Нечаев А. А., "Псевдослучайные и полилинейные последовательности.", Труды по дискретной математике, 1 (1997), 139-202.

3. М. М. Глухов, И. А. Круглов, А. Б. Пичкур, А. В. Черемушкин, Введение в теоретико-числовые методы криптографии, Лань, СПб., 2011, 394 с.

4. Р. Рокафеллар, Выпуклый анализ., Мир, Москва, 1973, 472 с.

Статья поступила 15.01.2014. 